\title{
La violencia urbana como fenómeno multicausal: un estudio en tres comunas de la ciudad de San Juan de Pasto
}

\author{
Urban violence as a multi-causal phenomenon: a study in \\ three communes of the city of San Juan de Pasto
}

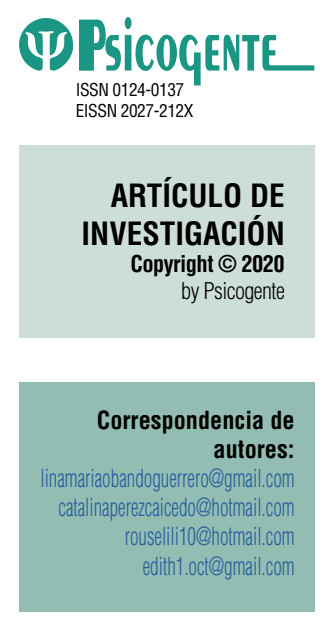

Recibido: 06/04/19 Aceptado: $14 / 08 / 19$ Publicado: 01/01/20

Lina María Obando Guerrero iD - Catalina Pérez Caicedo iD - Rosa Liliana
Cuastumal Meneses (iD - Edith de Lourdes Hernández Narváez iD
Universidad de Nariño, Nariño, Colombia

\section{Resumen}

Objetivo: Describir y comprender los factores socioambientales de la violencia urbana en tres comunas de la ciudad de San Juan de Pasto (Colombia).

Método: Investigación cualitativa en la que participaron 47 estudiantes, entre los 11 y 17 años (26 mujeres y 21 hombres) de tres Instituciones Educativas públicas, 16 docentes de diferentes asignaturas y 5 agentes comunitarios clave. El estudio se desarrolló en tres comunas con estrato socioeconómico bajo, de la ciudad de San Juan de Pasto-Colombia. La información se obtuvo mediante grupos focales y entrevistas semiestructuradas.

Resultados: La violencia urbana es un fenómeno multicausal atravesado por diferentes factores como, el abandono del Estado a las comunidades más vulnerables, la inequidad en la oferta de oportunidades de desarrollo para sus habitantes, el estigma y la exclusión propiciados por formas de organización social ilegales como las pandillas, las cuales pese a que satisfacen ciertas necesidades en los jóvenes, también favorecen comportamientos criminales y ejercen un control territorial inadecuado. Finalmente, las dinámicas familiares disfuncionales como el maltrato, la falta de apoyo familiar y la ausencia de los padres, son factores de riesgo que al interactuar con otros, contribuyen a la consolidación de la violencia urbana.

Conclusiones: Las intervenciones frente a la violencia urbana deben garantizar la presencia del Estado en las comunidades afectadas, mediante el despliegue de políticas públicas y programas sociales que propendan por mejorar las condiciones socioambientales y el empoderamiento. Se requiere impulsar estrategias para mejorar la seguridad y la convivencia ciudadana, proveer de mejores oportunidades de empleo y de educación formal y no formal en las que se incluyan también a las familias, garantizando el desarrollo integral de las comunidades, especialmente las más vulnerables, las cuales han sido afectadas sensiblemente por la violencia.

Palabras clave: factores socioeconómicos; organización social; control territorial y violencia urbana.

Abstract

Objective: Describe and understand the socio-environmental factors of urban violence in three communes of the city of San Juan de Pasto (Colombia).

Method: Qualitative research in which participed 47 students, between 11 and 17 years old (26 women and 21 men) from three public educational institutions, 16 teachers from different subjects and 5 key community agents. The study was carried on in three communities with a low socioeconomic status, from the city of San Juan de Pasto, Colombia. The information was obtained through focus groups and semi-structured interviews.

Results: Urban violence is a multi-causal phenomenon conditioned by different factors such as the abandonment of the State to the most vulnerable communities, the inequality in the offer of development opportunities for his inhabitants, the stigma and exclusion fostered by illegal forms of social organization like gangs, which, although they satisfy certain needs in young people, also favor criminal behavior and exercise inadequate territorial control. Finally, dysfunctional family dynamics such as abuse, lack of family support and the absence of parents are risk factors that, when interacting with others, contribute to the consolidation of urban violence.

Conclusions: Interventions against urban violence must ensure the presence of the State in the affected communities, through the deployment of public policies and social programs that tend to improve socio-environmental conditions and empowerment. It is necessary to promote strategies to improve security and

Cómo citar este artículo (APA):

Obando Guerrero, L.; Pérez Caicedo, C.; Cuastumal Meneses, R. \& Hernández Narváez, E. (2020). La violencia urbana como fenómeno multicausal: un estudio en tres comunas de la ciudad de San Juan de Pasto. Psicogente 23(43), 1-26. https://doi.org/10.17081/psico.23.43.3269 


\section{INTRODUCCIÓN}

En los últimos años se ha evidenciado que surge un nuevo tipo de violencia relacionada con el narcotráfico, el crimen organizado, el secuestro y el pandiIlismo como resultado de prácticas sociales y manifestaciones no resueltas entre diferentes sujetos o grupos (Dávila, 2016). Esta violencia denominada violencia urbana se ha establecido como tema de mayor atención pues la intranquilidad, el miedo, el temor y el desasosiego se han apoderado de la mayoría de los habitantes de las principales ciudades (Moreno, 2014). Según León (2015), es definida como aquellos actos criminales que se producen en las ciudades en el contexto de las relaciones sociales entre los individuos, la cual se da como consecuencia del rápido proceso de crecimiento y modernización de las zonas urbanas.

La violencia urbana se presenta con mayor frecuencia en aquellas zonas periféricas que deben afrontar problemáticas de accesibilidad y carencia de servicios, desintegración social y dificultad para la inserción laboral y política; además del empobrecimiento, la exclusión y la segregación residencial (Sabatini \& Brain, 2008). En Colombia, la violencia urbana en sectores populares o periféricos adquiere mayor magnitud a partir de la instalación de grupos armados dedicados a la realización de actividades ilegales, cuyo orden de poder repercute en la socialización y subjetivación de la comunidad, con mayor impacto en la población juvenil (Perea, 2002).

\subsection{Elementos teóricos y/o conceptuales}

Díaz y Esteves (2017) relatan que las expresiones de la violencia urbana incluyen diversos tipos de delitos (robo, asalto), actos violentos y criminales, comercio y consumo de narcóticos, en los espacios públicos como escenario. Esto ha conllevado a una lógica de urbanismo que blinda y segrega a las ciudades, dado que la espacialidad colectiva se ha abandonado, las sociedades se encuentran militarizadas y los comportamientos antisociales y negativos son una constante.

A partir de lo expuesto, se puede plantear que la representación social de la violencia urbana tiene un carácter hiperreal que conlleva a la existencia de una realidad material concreta que se expande simbólicamente invisi- 
bilizando otras situaciones relevantes como el aspecto social, económico y político. Estas construcciones sedimentan nociones que orientan diferentes intervenciones públicas basadas en una jerarquía y mandato impuesto por ciertos actores (Baudrillard, 1993), factor que influye en la exclusión de los sectores populares, que sumado a la segmentación territorial y la fragmentación socioeconómica aportan a la consolidación de una desigualdad simbólica en la cual dichos sectores ven limitadas sus posibilidades en la participación política y social (Duarte, 2017).

El estudio de la violencia urbana permite pensar este fenómeno como multicausal y multidimensional, puesto que su estructura se encuentra mantenida por diferentes factores socioambientales que en su relación influyen directamente en los procesos que la conforman. Dentro de los factores mencionados y que se analizarán en el presente artículo se encuentran los factores políticos, factores socioeconómicos, factores relacionados con el control territorial y factores familiares.

La estructura de las ciudades ha generado que el Estado oriente su atención hacia las zonas centrales dejando a un lado las zonas periféricas que en su mayoría son aquellas que presentan bajos recursos económicos (Hernández, Meneses \& Moreno, 2016) y donde se interpreta que el papel del Estado no es suficiente para dar solución a las problemáticas que se presentan, es por ello que en relación a los factores políticos, se crean e imponen nuevas reglamentaciones acordes a las necesidades de la comunidad, las cuales regulan y controlan el comportamiento de los miembros (Murcia, 2015).

Respecto a los factores socioeconómicos, en esta zona se evidencian fenómenos que confluyen tales como, el empobrecimiento y la desigualdad, la estigmatización y la segregación territorial, los cuales a su vez están ligados al desempleo, la precariedad laboral, la falta de oportunidades educativas, la deserción escolar y el fácil acceso a las armas de fuego (Briceño, 2002). Tras la falta de oportunidades y ante la necesidad de solventar los medios de supervivencia, se acude a nuevas fuentes económicas como es el comercio ilícito que la mayoría de veces está relacionado con el microtráfico (Murcia, 2015), surgiendo así un tercer factor de violencia urbana como es el control territorial por parte de grupos como pandillas, las cuales mantienen el poder, usando como recursos principales el miedo y la intimidación para establecer un nuevo orden social basado en la imposición de leyes y normas que afectan directamente el uso de los espacios públicos y el tejido social por el temor, la desconfianza e inseguridad que se genera en la comunidad (Lunecke, 2012). 
La génesis de las pandillas también encuentra explicación por un último factor que atraviesa la violencia urbana y es el familiar, cuyas dinámicas y estructura pueden precipitar, agravar o mantener conductas violentas, agresivas o antisociales (Sánchez, 2008). Como parte de los elementos de riesgo que inciden en este tipo de conductas, se encuentran el lugar donde está asentada la familia (como es la comunidad próxima en la que coexisten) delincuencia, violencia, pares que incitan a la conducta antisocial y consumo de SPA, conflictos maritales, interacciones coercitivas entre padres e hijos y pobres vínculos afectivos; modelos intergeneracionales de conductas violentas, pautas de crianza basadas en el castigo y el maltrato, así como actitudes pasivas, incoherencia en las normas, falta de consistencia y tratos inequitativos entre los hijos (Arias, 2013).

En lo que se refiere a los estudios que se han realizado en torno a la violencia urbana se resalta a Arias (2018), quien evidencia cómo esta trae consigo la segregación espacial y con ello la desigualdad en relación a la distribución económica de una ciudad y país, que conllevan a la expansión de una violencia producida por la falta de oportunidades laborales, sociales, económicas y educativas, viéndose afectada principalmente la población juvenil; se encuentra además que, la violencia urbana desde la perspectiva de los estudiantes se asocia con problemáticas como la exclusión, marginalidad, criminalidad y segregación. Asimismo, Jiménez y Pardo (2017) tras su investigación sobre la convivencia ciudadana y su relación con la violencia urbana, hacen énfasis en la necesidad de creación de políticas orientadas a la promoción de empleo, con el fin de mejorar las condiciones de vida de las comunidades, teniendo como fin la disminución de los niveles de pobreza y generando así un entorno propicio para reducir los niveles de violencia y conflictividad.

Camacho y Guzmán (1990) demuestran cómo en el desarrollo de la violencia urbana en las principales ciudades de Colombia como Cali y Medellín, se han generado diferentes formas de expresión de esta que denotan una instrumentalidad, es decir, no únicamente se encuentran actores e intereses que están en juego en el desarrollo de la violencia, sino que también se da una racionalización del "uso y recurso de la violencia de tal manera que se sabe en qué circunstancias utilizarla, con qué objetivos y los procedimientos más adecuados para ponerla en práctica" (p.47).

A nivel regional Narváez y Pérez (2018) realizaron un estudio en la Comuna 10 donde describen cómo la violencia urbana y su naturalización conllevan 
a procesos de identificación intergeneracional que permiten la reproducción de "patrones de sociabilidad violenta, ligados a la práctica delictiva" ( $p$. 78). Los grupos de jóvenes por medio de su incursión en prácticas ilegales, generan dentro de la comunidad un temor generalizado que sobrelleva un aumento en los niveles de inseguridad a través de la realización de actividades ilegales y criminales. De igual manera, se observa cómo la dinámica social de la comuna se ve envuelta por la emergencia de grupos ilegales que por medio de su control social y territorial convierten los espacios barriales en escenarios de conflicto que conllevan a la contradicción entre espacios transitables para la comunidad y las fronteras imaginarias que son impuestas por estos actores (Narváez, 2013).

A partir de lo anterior se reconoce que la violencia urbana al ser multicausal conlleva diferentes consecuencias; por ello surge el interés de describir los factores socioambientales relacionados con este fenómeno en tres comunas de la ciudad de San Juan de Pasto, dado que, al identificarlos se puede establecer una línea base que permite la descripción de cada uno de los componentes de la violencia urbana y las interacciones que hay entre ellos para reconocer su funcionamiento, y de esta manera establecer un marco tanto conceptual como práctico que tenga uso en futuras investigaciones en donde se desarrollen planes de intervención cuyas estrategias estén encaminadas al mejoramiento de las condiciones de seguridad y convivencia ciudadana.

Se debe tener en cuenta que, las comunas objeto de estudio se caracterizan por presentar altos niveles de violencia urbana, según la Encuesta de Convivencia y Seguridad Ciudadana -ECSC (Departamento Administrativo Nacional de Estadísticas- DANE, 2017) San Juan de Pasto es una de las ciudades que presentó en el año 2016 una tasa de victimización del 23,9\%, acompañada con un alto nivel de percepción de inseguridad por parte de sus habitantes (DANE, 2017).

Por otra parte, la población objeto de estudio se caracteriza por presentar altos índices de pobreza, lo cual se relaciona con la situación actual del departamento de Nariño que, según el DANE (2018), para el año 2017 los índices disminuyeron pasando del $45,7 \%$ al $40,2 \%$, sin embargo, se evidencia que continúa siendo una problemática a tratar. Estos aspectos junto con la desigualdad social (Salas, 2010), las dinámicas familiares conflictivas, el poco acceso a empleo y educación repercuten en el incremento de la violencia urbana en las comunas objeto de estudio y generan que gran parte de la 
población juvenil al terminar sus estudios secundarios se vincule a pandillas dedicadas principalmente al hurto y al consumo de sustancias psicoactivas (Bernal, 2013).

\section{MÉTODO}

\subsection{Diseño}

El presente estudio se enmarca en el paradigma cualitativo, el cual se caracteriza por analizar de manera individual y concreta un fenómeno por medio de la comprensión o interpretación de los significados intersubjetivos de la acción social (Rivas, 2011). Este se ocupa de los aspectos que son subjetivamente aprehensibles por medio de un análisis de aquellos procesos que no son susceptibles de ser medidos en términos de frecuencia (Izcara, 2014). En esta lógica, se buscó identificar y comprender los factores socioambientales que mantienen la violencia urbana en tres comunas de la ciudad de Pasto.

El método empleado fue la fenomenología que se caracteriza por comprender las dinámicas de los sujetos a partir de las experiencias vividas en su contexto social, lo cual les permite construir un significado en común (Guerrero, Prado, Kempfer \& Ojeda, 2017). De esta manera, la presente investigación se desarrolla a partir de las percepciones y opiniones que tienen los sujetos objeto de estudio de la violencia urbana.

\subsection{Participantes}

La unidad de análisis estuvo conformada en su mayoría por 47 adolescentes ( 26 mujeres y 21 hombres) entre los 11 y 17 años. Como criterios de inclusión se tuvo en cuenta que estos estuviesen inscritos en las tres Instituciones Educativas pertenecientes a tres comunas de la ciudad de Pasto, Colombia, asimismo, debían tener debidamente diligenciado el asentimiento y consentimiento informado. De igual manera, participaron 5 agentes clave quienes debían trabajar en los colegios y vivir en las comunas en mención.

\subsection{Instrumentos}

Para la recolección de la información se emplearon los protocolos y los formatos de preguntas correspondientes a las técnicas de grupo focal y entrevista semiestructurada (Ver Anexos 1 y 2 ). 
El grupo focal es una herramienta que posibilita el diálogo e intercambio de ideas y pensamientos frente a una situación o problemática, a partir de una dinámica en la que los sujetos develan sus experiencias cotidianas y estas pueden ser confirmadas o refutadas por los otros participantes (Silvera, Colomé, Heck, da Silva \& Viero, 2015). A partir de la aplicación de cinco grupos focales se dio paso a la interpretación y observación de las reacciones tanto grupales como individuales de los participantes frente a las problemáticas planteadas en razón de las subcategorías propias de la investigación.

En segundo lugar, se procedió a la aplicación de entrevistas semiestructuradas con agentes claves de la comuna. A partir de la definición de Díaz, Torruco, Martínez y Varela (2013) se entiende esta técnica como un elemento que posibilita la adaptación a los participantes, puesto que permite el ajuste de las preguntas y la flexibilidad en el desarrollo de la entrevista, aspecto que genera un ambiente de motivación en cuanto a la aclaración de términos, ambigüedades y la reducción de formalismos por parte del entrevistador. Se construyeron diferentes preguntas orientadoras cuyo objetivo se basa en la identificación de las realidades subjetivas de los participantes mediante el diálogo continuo.

En cada técnica se incluyeron subcategorías deductivas, construidas a partir de la revisión de literatura científica sobre la categoría violencia urbana en América Latina, a nivel nacional, regional, y específicamente en las comunas de estudio, valorando además, la observación independiente previamente realizada por parte del equipo de investigación en dichos contextos.

En consecuencia, se establecieron, de la forma más concreta y precisa posible de acuerdo a la realidad de investigación, las subcategorías de factores políticos, socioeconómicos, relacionados con el control territorial y factores familiares. Las preguntas de cada subcategoría deductiva y su totalidad en cada técnica fueron evaluadas por jueces expertos a partir de criterios de calificación tales como claridad, pertinencia, relevancia, redundancia y suficiencia. De esta manera, con la concordancia interpretativa del fenómeno entre jueces e investigadoras, la adecuada reconstrucción y consenso de las múltiples realidades percibidas y construidas por los sujetos de estudio y la congruencia de la relación teoría y realidad, se logró comprender la violencia urbana desde una perspectiva multifactorial, cumpliendo paralelamente con aspectos de validez y confiablidad científica requeridos en los procesos de 
investigación, para este caso desde el paradigma cualitativo (Cortés, 1997; Martínez, 2006).

\subsection{Procedimiento}

Para llevar a cabo el estudio, en primer lugar, se solicitó autorización de ingreso a las diferentes Instituciones Educativas presentándoles el proyecto de investigación y la metodología de trabajo propuesta. Posteriormente, se tuvo el primer contacto con los estudiantes y agentes clave con quienes se desarrolló el proceso de consentimiento informado procediendo a realizar los grupos focales y entrevistas semiestructuradas. Finalmente, se llevó a cabo el análisis y discusión de los resultados obtenidos.

\subsection{Análisis de datos}

El análisis de la información se realizó a través de las siete fases interactuantes propuestas por Cisterna (2005), que incluyen la categorización apriorística, selección de la información, triangulación de la información por cada estamento, triangulación entre estamentos, triangulación entre las diversas fuentes de información, triangulación con el marco teórico e interpretación de la información. Para la categorización apriorística, se organizó la información, a partir de la categoría general de estudio, factores socioambientales de la violencia urbana, se identificaron y diferenciaron las distintas subcategorías a saber, factores socioeconómicos, factores políticos, factores relacionados con el control territorial y factores familiares. En esta fase se tuvieron en cuenta dos criterios consecutivos, la pertinencia y la relevancia.

Ahora bien, con respecto a la triangulación de la información por cada estamento, con el procedimiento inferencial se contruyeron conclusiones ascendentes, agrupando las respuestas relevantes por tendencias, según la coincidencia o divergencia en cada uno de los instrumentos aplicados, en un proceso de diferentes niveles de análisis desde el cruce de los resultados por cada subcategoría (conclusiones de primer orden), el cruce de las conclusiones de primer nivel agrupándolas por su pertenencia a la categoría central (conclusiones de segundo nivel), y el cruce de las conclusiones categoriales por cada estamento (conclusiones de tercer nivel).

Consecutivamente, la triangulación de la información entre estamentos permitió establecer relaciones de comparación significativas entre los 
diferentes grupos de sujetos indagados, en función de los diversos tópicos interrogados desde un carácter general y otro específico. En el carácter general la comparación significativa se hizo desde las conclusiones de tercer nivel, triangulando la opinión de los estamentos a las preguntas centrales de la investigación. En el carácter específico las comparaciones tuvieron lugar desde las subcategorías de cada estamento.

Para la triangulación entre las diversas fuentes de información, se tuvieron en cuenta las conclusiones de segundo y tercer nivel, que integraran la triangulación interestamental por cada instrumento aplicado, propiciando nuevos procesos interpretativos. Además, en la triangulación con el marco teórico, se generó una discusión entre la literatura especializada, actualizada y pertinente sobre las diferentes subcategorías de investigación y lo que se encontró en la indagación de campo. Y finalmente, la interpretación de la información integró los hallazgos en su totalidad que develaron los factores socioambientales que mantienen la violencia urbana en tres comunas de la ciudad de Pasto.

\section{RESULTADOS}

El análisis de la información permitió identificar que la violencia urbana es un fenómeno atravesado por factores que hacen parte tanto de los micro como de los macrocontextos de la vida comunitaria. En esta sección se presentan los hallazgos más relevantes que dan cuenta de las comprensiones alcanzadas como producto del análisis de la información desde las subcategorías planteadas para cada estamento.

\subsection{Factores políticos}

En las comunas objeto de estudio, se evidenció que existe una alta percepción de abandono por parte del Estado, lo que ha traído consecuencias políticas y económicas determinantes en la organización y dinámica de las comunidades. A nivel político, los participantes perciben un abandono estatal por lo que optan por buscar estrategias de protección y defensa grupal, haciendo uso de herramientas propias como la "limpieza social" y campañas de autocuidado, refiriendo: "entre los vecinos se ponen un aviso y salen encapuchados para tener seguridad en el barrio, salen con palos así desde las 9, 10, 11 hasta las 2 por ahí y van a buscar a los muchachos que venden droga para evitar que lo sigan haciendo". A pesar de que la finalidad de las dinámicas 
mencionadas es la erradicación del comercio de SPA, estas incrementan la percepción de que se puede gestionar la justicia a manos propias, reivindicando la ausencia del Estado y favoreciendo que los niveles de violencia urbana incrementen progresivamente puesto que se utiliza una estrategia basada en la agresión e intimidación entre los propios habitantes e incluso los agentes del Estado, quienes según las comunidades participantes abusan del poder y muchas veces incurren en violencia y maltrato para garantizar el orden.

\subsection{Factores socioeconómicos}

En este aspecto se encuentra que los sujetos perciben que son estigmatizados y excluidos por la sociedad, refiriendo: "piensan que acá es un barrio, digamos que vienen y ya lo van robando y pues no es asi'. Se observa que estos procesos han conllevado a que la mayoría de trabajos sean informales (carpintería, mototaxismo, comercio en la calle, entre otros) o relacionados con actividades económicas ilegales (venta de SPA, robos, extorsiones). Este aspecto ha generado que los jóvenes, al tener recursos económicos limitados para acceder a la educación, sean vulnerables a vincularse a este último tipo de actividades y opten por otros caminos que fomentan los niveles de violencia barrial.

Aunque la estigmatización no es generalizada, las características de las comunas como la existencia de lugares poco transitados, la lejanía respecto a sectores centrales de la ciudad y la pertenencia a estratos socioeconómicos bajos, han favorecido comportamientos criminales que aunque no sean realizados por la totalidad de la población, socialmente son tildados de "peligrosos".

En cuanto a la economía ilegal, de acuerdo con los participantes, el microtráfico se condensa con el establecimiento de pandillas, quienes buscan caracterizarse de formas particulares y control territorial sobre ciertas zonas de las comunidades para adquirir mayor dominio y poder respecto a otros grupos y campo en el comercio de SPA. Un agente clave refiere: "Ias pandillas en general se reúnen en esquinas donde consumen sustancias psicoactivas y arman problemas, hacen que a uno le de miedo salir". El miedo, la inseguridad y la zozobra al hacer parte de la cotidianidad de estas comunas se constituyen en mantenedores de la violencia urbana (Ver Figura 1). 


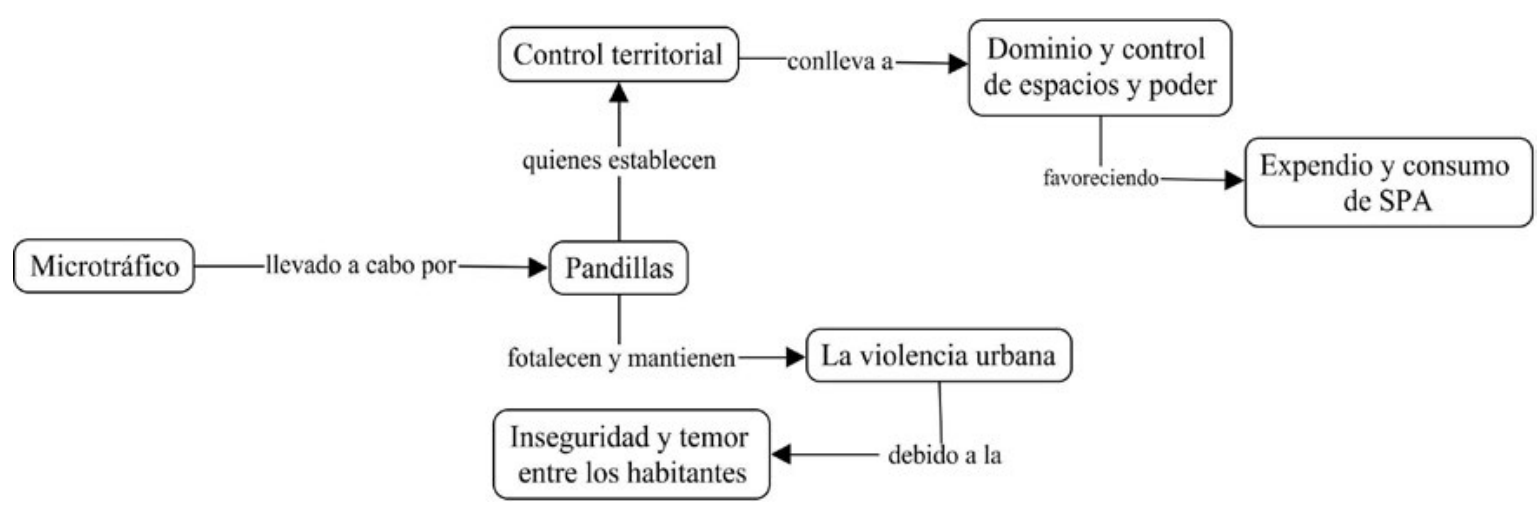

Figura 1. Estructuras y dinámicas que fortalecen y mantienen la violencia urbana

\subsection{Factores relacionados con el control territorial}

La organización de pandillas es un factor común en las tres poblaciones estudiadas. Los jóvenes que pertenecen a las pandillas necesitan ser identificados y "respetados" en la comunidad y por ello caracterizan su manera de vestir, caminar, hablar y comportarse, es así como utilizan vestimentas con una señal particular, habitan en lugares estratégicos como una esquina, las gradas o callejones; lugares que están ligados al territorio dado que actúan como señales que resultan ser de gran importancia especialmente cuando están relacionadas con el microtráfico mediante el cual se disputa el nivel y jerarquización de las pandillas, es decir, el grupo con mayor poder y dominio territorial es aquel que tiene más campo para el consumo, expendio y tráfico de drogas; es por ello que los jóvenes vinculados al negocio, imponen miedo y temor entre los habitantes. Los participantes refieren: "en ciertas zonas como canchas o callejones es mejor no pasar en horarios después de las 7 u 8 de la noche porque uno está en riesgo". Estas condiciones refuerzan la estigmatización que existe frente a las comunas como zonas de alto riesgo y favorece la naturalización de la violencia como mecanismo de control y regulación, dando paso a la evitación por parte de los habitantes quienes prefieren no hacer frente a estas dinámicas, cuidarse y mantener a salvo a sus familias.

Como hallazgo emergente se identificó que las pandillas representan un escenario ideal como satisfactor de distintas necesidades de los jóvenes vulnerables que hacen parte de ellas (Figura 2): 


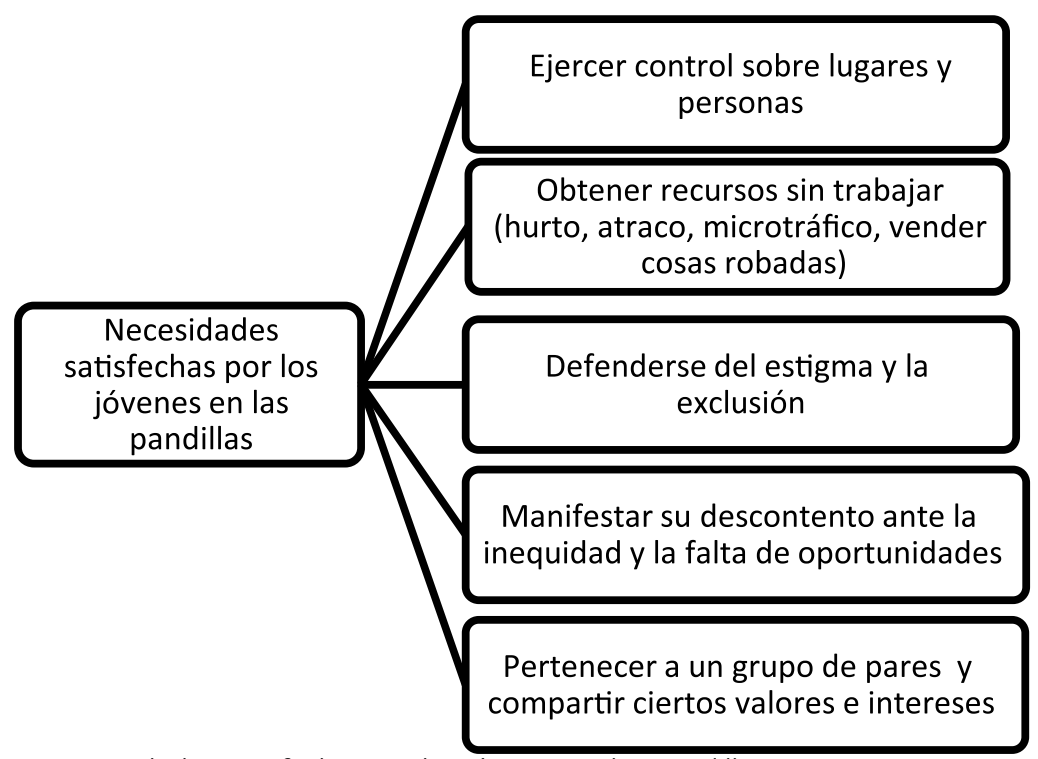

Figura 2. Necesidades satisfechas por los jóvenes en las pandillas

\subsection{Factores familiares}

En las comunas, se evidencia que hay problemáticas en el funcionamiento familiar que alteran la interacción de los miembros, convirtiéndose en un factor de riesgo frente a la violencia. Así, los participantes refieren que existe la dificultad para establecer roles lo que afecta el ejercicio de la autoridad por parte de los padres. Además, se identifican interacciones agresivas entre los miembros del hogar donde se presenta la violencia física y psicológica en diferentes manifestaciones.

Los adolescentes entrevistados relatan la falta de acompañamiento por parte de sus padres quienes dedican gran parte del tiempo al trabajo dejando a sus hijos sin compañía u orientación confiable, aspecto que conlleva a que muchos de ellos no perciban apoyo emocional en sus familias. Un factor común en los grupos participantes es la utilización de la violencia como una herramienta para la crianza de los hijos, pauta que se ha naturalizado en la cotidianidad y que se legitima con la práctica constante, creando y recreando imaginarios en los cuales se relaciona castigo con amor y se equipara violencia con formas de cuidado y protección.

En el siguiente esquema se encuentran algunos relatos que evidencian problemas familiares estructurales favorecedores de distintas formas de violencias (Ver Figura 3). 

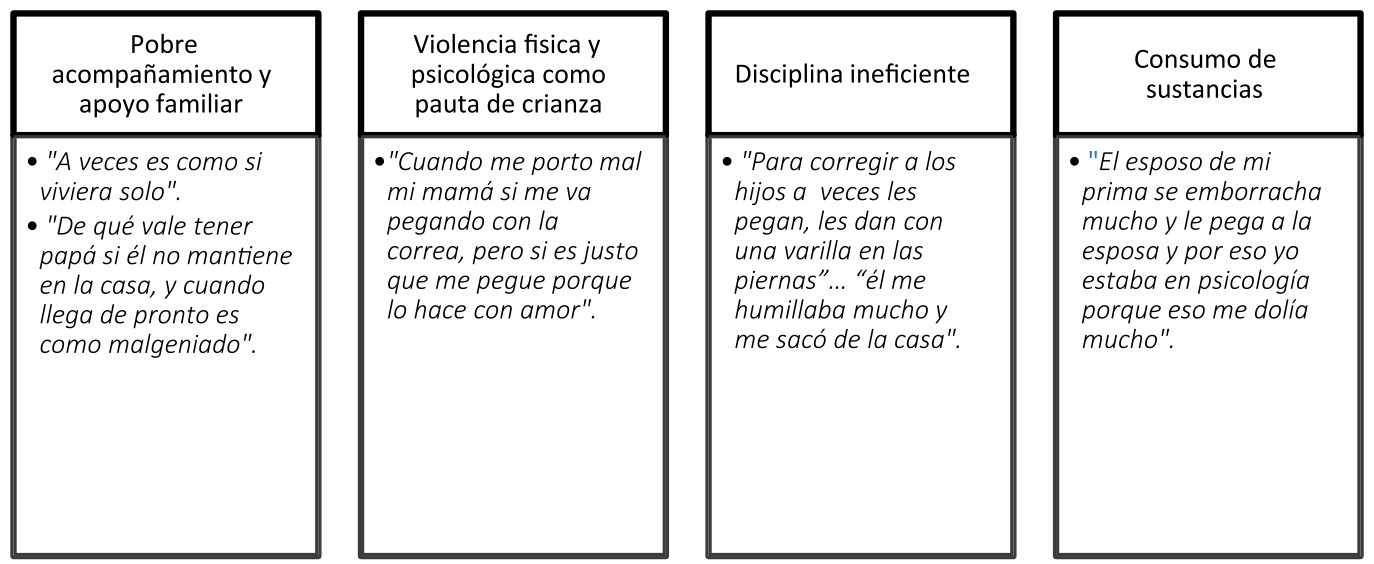

Figura 3. Problemas en la dinámica familiar que favorecen la violencia

\section{DISCUSIÓN}

El estudio tuvo como objetivo describir y comprender los factores socioambientales de la violencia urbana en tres comunas de la ciudad de San Juan de Pasto. Se parte de la idea de la violencia como un fenómeno multidimensional, asumiéndolo como fenómeno difícil de realizar su abordaje, análisis y comprensión, por lo cual se recurre a desglosar los factores que lo componen para así posteriormente establecer sus interrelaciones (Colombara, 2011). Inicialmente se plantea que, la percepción de los habitantes frente a la ausencia del funcionamiento del Estado al momento de regular acciones violentas y delictivas es determinante para las dinámicas políticas que se dan al interior de las comunas (Frühling \& Sandoval, 1998), aspecto confirmado por los sujetos de estudio, quienes a partir de la percepción de abandono estatal no denuncian las actividades ilícitas e ilegales que se desarrollan dentro de las comunidades, prefiriendo desarrollar estrategias de autoprotección y defensa grupal que las interpretan como campañas de autocuidado.

En estos contextos donde se presentan dificultades de presencia estatal y policial, las organizaciones alternativas de orden social se instalan y se naturalizan en las comunidades, acrecentado la violencia urbana (Hernández, et al., 2016), también reforzada por el factor socioeconómico, en el que se evidencia la estigmatización territorial catalogada por Kessler y Dimarco (2013) como el proceso por el cual un determinado espacio queda reducido a ciertos atributos negativos que aparecen magnificados, estereotipados, produciendo como resultado su devaluación o desacreditación social. Para Fuentes y Hernández (2013), la exclusión está determinada por procesos económicos, políticos y culturales, identificados respectivamente en la 
exclusión tanto de bienes y servicios básicos como en la participación en la toma de decisiones políticas. Son estas características, las mismas que están presentes en la nueva pobreza urbana, sumadas al deterioro del tejido social y la carente participación y relaciones vecinales.

En efecto, a partir de violencias invisibles como la exclusión y la estigmatización territorial, los jóvenes de las comunas estudiadas sufren de forma particular las consecuencias de los límites para acceder a un trabajo formal, a la educación y a vínculos sociales formales externos, aspectos que estimulan la adhesión a economías ilegales para conseguir un sustento económico (Linares, 2013). Además, se podría decir que las conductas que se presentan en las comunas estudiadas (microtráfico, vandalismo, robos, consumo de SPA) reflejan la inequidad promovida por el mismo Estado, en la distribución de bienestar hacia toda la población, pues los recursos y oportunidades no se dan de acuerdo a las necesidades y características de las comunidades (Dammert, 2005).

Según Tijoux (1995), los jóvenes son los principales afectados por una mayor tasa de desempleo sectorial, una mayor presión competitiva, una decreciente proyección a identidades colectivas y una curva ascendente de actitudes anómicas. A partir de lo anterior, se concluye que la incapacidad de la estructura social para proveer a los individuos de los recursos necesarios para lograr metas tanto laborales como educativas y económicas, es una de las principales razones por las cuales los adolescentes o jóvenes se adhieren a estructuras delincuenciales como las pandillas que brindan solvencia de los elementos anteriormente mencionados, así mismo, Tullock (1971, citado en Cuartas, Valencia \& Zapata, 2011) plantea por medio de su escuela económica del crimen que los crímenes se dan debido a la relación entre la existencia de una oferta y demanda que conllevan a la realización de ciertos comportamientos delictivos, que se ven incentivados por la relación costo-beneficio. Este aspecto es referido por los participantes quienes afirman que la existencia de grupos se conforma principalmente por adolescentes, los cuales se caracterizan por realizar actividades ilegales o delictivas, como método para la subsistencia económica.

De igual manera, las pandillas buscan establecer un nuevo orden social basado en la imposición de leyes y normas, empleando mecanismos como el miedo y la intimidación quedando manifiesto el control territorial que explica la relación de los grupos ilegales con el microtráfico, por medio de la resignificación del territorio como fuente de abastecimiento e identidad, el cual 
deberá ser defendido y preservado como aquel resguardo y proveedor de los beneficios obtenidos mediante la venta y consumo de SPA (Murcia, 2015). De acuerdo con Salazar y Frasser (2013), el control territorial corresponde a una disputa del mercado de las drogas entre diversos grupos que son los encargados de accionar en la defensa del control de los expendios locales de droga denominados "ollas".

Por último, las dinámicas familiares ejercen una influencia significativa en el comportamiento de los individuos, dado que es ahí donde se aprenden valores, conductas y modos de vida que se suelen replicar en otros contextos como el escolar, el comunitario y el laboral. De esta manera, se encuentra que las debilidades en el núcleo familiar precipitan la conformación de pandillas que muchas veces están constituidas por jóvenes que viven una constante violencia intrafamiliar. Este postulado es reafirmado por Arias (2013) quien apunta a que las falencias en las dinámicas familiares relacionadas con el establecimiento de roles, la violencia física y psicológica, pobres vínculos afectivos, actitudes pasivas e incoherencia entre lo que se dice y se exige, se constituyen en factores de riesgo para precipitar, agravar o mantener conductas violentas o antisociales. A esto Suárez y Jama (2015) añaden que la falta de acompañamiento es determinante en la decisión que toman los jóvenes de emplear su tiempo libre y realizar o no conductas delictivas.

Las dinámicas familiares disfuncionales sumadas al contexto barrial caracterizado por dificultades económicas y segregación residencial devienen en el riesgo de adhesión a grupos ilegales, los cuales por medio del aprendizaje social y socialización de conductas violentas, visibilizan aspectos de semejanza en torno a modos de vida similares y trayectorias entre sus miembros, es así como la juventud encuentra en las pandillas cohesión y sentido de pertenencia a través de la realización de actividades ilegales o violentas y el consumo de SPA y la apropiación y defensa del territorio en los enfrentamientos entre grupos rivales.

\section{CONCLUSIONES}

La percepción de ausencia del Estado se asocia con la consolidación de nuevas estructuras y dinámicas políticas al interior de las comunas estudiadas, que si bien buscan la protección y defensa grupal ante la ausencia del sistema penal, terminan incrementando la violencia urbana pues se basan en la agresión e intimidación. 
Con respecto a lo socioeconómico, la percepción de segregación y estigmatización por la sociedad ha traído como consecuencia la dificultad en el acceso al campo laboral y académico razón por la cual se realizan trabajos informales y se generan vinculaciones en actividades ilegales relacionadas con el narcotráfico y el hurto, incrementando la violencia.

La actividad económica relacionada con el microtráfico es mantenida por las pandillas ilegales que constantemente se disputan el control territorial, el poder y el reconocimiento como grupo y como comercializador de SPA. En la formación de pandillas también incide la estructura y dinámica familiar, observándose debilidades en el establecimiento de roles y autoridad, la comunicación y el acompañamiento a los hijos en etapas críticas de su desarrollo como la niñez y la adolescencia.

Si bien los problemas encontrados al interior de las familias no son la causa directa de la violencia urbana, su interacción con otros factores facilitan las condiciones para que los adolescentes se sientan solos, tengan pobre apoyo emocional, experimenten poca pertenencia en su familia y esto los convoca a involucrarse en actividades vinculadas con comportamientos ilegales $y$ violentos.

Para finalizar, el presente estudio permite visualizar la necesidad de enfocarse en la educación formal e informal a sumiéndola como un proceso generador de nuevas ciudadanías en torno a la ética y la moral, la solución consensuada de conflictos, las prácticas deliberativas y la democracia en su sentido amplio, de la mano de proyectos que promuevan actividades alternativas que fortalezcan en la juventud el desarrollo personal, académico y económico, previniendo su vinculación a grupos de riesgo como las pandillas, para lo cual también se requiere el apoyo estatal. Además, es necesario formular e implementar programas de psicoeducación para mejorar la comunicación asertiva y el apoyo emocional al interior de los hogares, esto con el fin de que la familia sea un factor protector y no de riesgo.

Agradecimientos: A las Instituciones Educativas, estudiantes, docentes y agentes clave que participaron en la investigación. A la Universidad de Nariño y al grupo de Investigación Libres Pensadores.

Financiamiento: Artículo derivado de la investigación titulada "Factores socioambientales de la violencia urbana inmersos en los procesos de convivencia escolar de las Instituciones Educativas oficiales de las comunas 3, 7 y 10 de la ciudad de Pasto. Financiado por la Universidad de Nariño en la Convocatoria Docente 2016. 


\section{REFERENCIAS}

Arias, J.D. (septiembre 2018). Más allá de la violencia urbana: el territorio como oportunidad de formación ciudadana. En J.C. Ramos, A.C. Rodríguez, N. Moreno \& C. Zambrano (organizadores), Didáctica de las Ciencias Sociales: ¿Enseñar disciplinas o enseñar desde problemas sociales relevantes? Simposio llevado a cabo en el IX Encuentro de la Red Colombiana de Investigación en Didáctica de las Ciencias Sociales, Bogotá, Colombia.

Arias, W. (2013). Agresión y violencia en la adolescencia: la importancia de la familia. Unifé, 21(1), 23-34. http://ucsp.edu.pe/imf/wp-content/uploads/2014/12/ Agresionyviolenciaenlaadolescencia1.pdf

Baudrillard, J. (1993). El intercambio simbólico y la muerte. Caracas, Venezuela: Monte Ávila.

Bernal, R. (2013, marzo 23). Comuna 10. Zona de inseguridad en Pasto. Diario del Sur. http://diariodelsur.com.co/comuna-10-zona-de-inseguridad-en-pasto-34214

Briceño, R. (2002). La nueva violencia urbana de América Latina. Sociologías, 4(8), 34-51. http://www.observatoriodeseguranca.org/files/n8a03.pdf

Camacho, A. \& Guzmán, A. (1990). La violencia urbana en Colombia: síntesis de un estudio exploratorio en una ciudad colombiana. Boletín Socioeconómico, (20), 42-56. http://bibliotecadigital.univalle.edu.co/bitstream/10893/5491/1/ La\%20violencia\%20urbana\%20en\%20Colombia\%20Sintesis.pdf

Cisterna, F. (2005). Categorización y triangulación como procesos de validación del conocimiento en investigación cualitativa. THEORIA, 14(1), 61-71. https://www. redalyc.org/html/299/29900107/

Colombara, M. (2011). Violencia urbana. Su relación con la violencia de género. Revista Geográfica de América Central, 2(47), 1-16. https://www.redalyc.org/ articulo.oa?id=451744820315

Cortés, G. (1997). Confiabilidad y validez en estudios cualitativos. Educación y Ciencia, 1(1), 78-82. http://educacionyciencia.org/index.php/educacionyciencia/article/ download/111/pdf

Cuartas, D., Valencia, G.D. \& Zapata, O. (2011). Violencia y criminalidad urbana en Colombia (2000-2010): el enfoque económico ortodoxo. Perfil de Coyuntura Económica, (17), 141-164. http://www.scielo.org.co/pdf/pece/n17/n17a7.pdf

Dammert, L. (2005). Violencia criminal y seguridad ciudadana en Chile. Serie Políticas Públicas, 109. Santiago de Chile: CEPAL. http://www.eclac.org/publicaciones/ $\mathrm{xml} / 1 / 22061 /$ sps_109.pdf

Dávila, L. F. (2016). Violencia urbana, conflicto y crimen en Medellín: una revisión de las publicaciones académicas al respecto. Revista Criminalidad, 58(2), 107-121. http://www.scielo.org.co/pdf/crim/v58n2/v58n2a03.pdf

Departamento Administrativo Nacional de Estadísticas (2017). Encuesta de convivencia y seguridad ciudadana 2017. https://www.dane.gov.co/files/investigaciones/poblacion/convivencia/2017/Bol_ECSC_2017.pdf

Departamento Administrativo Nacional de Estadísticas (2018). Pobreza monetaria Nariño- Año 2017. https://www.dane.gov.co/files/investigaciones/condiciones_ vida/pobreza/2017/Narino_Pobreza_2017.pdf

Díaz, L., Torruco, U., Martínez, M. \& Varela, M. (2013). La entrevista, recurso flexible y dinámico. Investigación en Educación Médica, 2(7). http://riem.facmed.unam. $\mathrm{mx} /$ node/47

Díaz, C.E. \& Esteves, M. (2017). Violencia urbana e inseguridad en espacios de vida colectiva. Revista Brasileira de Estudos Urbanos e Regionais, 19(3), 440-458. https://www.redalyc.org/pdf/5139/513954274003.pdf 
Duarte, M. (2017). Favela, violencia urbana y políticas de seguridad pública en Río de Janeiro. Quid, (7), 174-194. https://publicaciones.sociales.uba.ar/index.php/ quid16/article/viewFile/2565/2254

Frühling, H. \& Sandoval. L. (1998). Percepciones de inseguridad y realidad delictual en tres comunas populares de Santiago. Estudios Públicos, (68), 254-306 https:// www.cepchile.cl/cep/site/artic/20160303/asocfile/20160303183847/rev68_ fruhling.pdf

Fuentes, C. \& Hernández, V. (2013). Segregación socioespacial y accesibilidad al empleo en Ciudad Juárez: Chihuahua (2000-2004). Región y sociedad, 25(56), 43-74. https://regionysociedad.colson.edu.mx:8086/index.php/rys/article/view/99

Guerrero, R.F., Prado, M., Kempfer, S. \& Ojeda, M.G. (2017). Momentos del Proyecto de Investigación Fenomenológica en Enfermería. Index de Enfermería, 26(1-2), 67-71. http://scielo.isciii.es/scielo.php?script=sci_arttext\&pid=S1132-12962017000100015

Hernández, E., Meneses, B. \& Moreno, N. (2016). La resiliencia comunitaria en contextos de violencia urbana. Revista de Psicología GEPU, 7(2), 24-46. https:// revistadepsicologiagepu.es.tl/La-Resilencia-Comunitaria-en-contextos-de-Violencia-Urbana.htm

Izcara, S.P. (2014). Manual de investigación cualitativa. Coyoacán, México: Distribuciones Fontamara.

Jiménez, A. \& Pardo, J. (2017). Violencia urbana y dinámica social en Cartagena de Indias: recomendaciones estratégicas para mejorar la convivencia ciudadana. Revista Internacional de Cooperación y Desarrollo, 4(1), 172-187. http://revistas. usbbog.edu.co/index.php/Cooperacion/article/view/3123/2613

Kessler, G. \& Dimarco, S. (2013). Jóvenes, policía y estigmatización territorial en la periferia de Buenos Aires. Espacio Abierto, 22(2), 221-243. https://ri.conicet.gov. ar/bitstream/handle/11336/5878/CONICET_Digital_Nro.6807_A.pdf?sequence=2\&isAllowed=y

León, J. (2015). Determinantes de la violencia urbana en Lima Metropolitana y Callao: Casos de delitos contra el patrimonio. Pensamiento Crítico, 20(1), 75-96. http://revistasinvestigacion.unmsm.edu.pe/index.php/econo/article/ view/11485/10331

Linares, S. (2013). Las consecuencias de la segregación socioespacial: un análisis empírico sobre tres ciudades medias Bonaerenses (Olavarría, Pergamino y Tandil). Cuaderno urbano, 14(14). https://ri.conicet.gov.ar/handle/11336/1159

Lunecke, G. (2012). Violencia urbana, exclusión social y procesos de guetización: la trayectoria de la población Santa Adriana. Revista Invi, (74), 287-313. http:// www.revistainvi.uchile.cl/index.php/INVI/article/view/604/1010

Martínez, M. (2006). Validez y confiabilidad en la metodología cualitativa. Paradigma, 27(2), 07-33. http://www.scielo.org.ve/scielo.php?script=sci_ arttext\&pid=S1011-22512006000200002

Moreno, A. E. (2014). Violencia delictiva e inseguridad ciudadana. Temas sociales, (35), 81-112. http://www.revistasbolivianas.org.bo/pdf/rts/n35/n35_a04.pdf

Murcia, W. (2015). Las pandillas en El Salvador. Propuestas y desafíos para la inclusión social juvenil en contextos de violencia urbana. Recuperado del sitio web CEPAL: https://repositorio.cepal.org/bitstream/handle/11362/39362/S1501050_es.pdf?sequence=1\&isAllowed=y

Narváez, J.H. (2013). Violencia barrial, la tensión social como crisis humanitaria. Tesis psicológica, 8(1), 56-67. https://www.redalyc.org/articulo.oa?id=139029198005 
Narváez, J.H. \& Pérez, C. (2018). Procesos de violencia urbana emergentes de la institución de fronteras imaginarias en entornos barriales de la comuna 10 de Pasto. Revista Virtual Universidad Católica del Norte, (54), 69-86. http://revistavirtual. ucn.edu.co/index.php/RevistaUCN/article/view/983/1432

Perea, C. (2002). Somos expresión, no subversión: juventud, identidades y esfera pública en el sur oriente bogotano. En H. Cubides, M. Laverde, C. Valderrama, (Eds.), Viviendo a toda. Jóvenes, territorios culturales y nuevas sensibilidades (pp.129-150). Bogotá, Colombia: Siglo de hombre editores

Rivas, F. (2011). La relevancia del paradigma cualitativo en las ciencias sociales: un análisis histórico descriptivo. Fermentum. Revista Venezolana de Sociología y Antropología, 21(61), 289-319. https://www.redalyc.org/pdf/705/70538663008. pdf

Sabatini, F \& Brain, I. (2008). La segregación, los guetos y la integración social urbana: mitos y claves. Revista Eure, XXXIV(103), 5-26. https://scielo.conicyt.cl/scielo. php?script=sci_arttext\&pid=S0250-71612008000300001

Salas, W. (2010, octubre 5). Para resolver la violencia urbana. El Colombiano. http://www.elcolombiano.com/historico/para_resolver_la_violencia_ urbana-FEEC_106928

Salazar, B., \& Frasser, C. (2013). Menos cocaína, más violencia. Revista de Economía Institucional, 15(29), 327-334. https://www.redalyc.org/pdf/419/41929178016. pdf

Sánchez, V. (2008). La influencia de las familias en las conductas antisociales de los jóvenes (tesis de pregrado). Universidad Pedagógica Nacional, Bogotá, Colombia. http://200.23.113.51/pdf/24977.pdf

Silveria, D., Colomé, C., Heck, T., da Silva N. \& Viero, V. (2015). Grupo focal y análisis de contenido en la investigación cualitativa. Index de Enfermería, 24(1-2). http:// scielo.isciii.es/scielo.php?script=sci_arttext\&pid=S1132-12962015000100016

Suárez, H. \& Jama, V. (2015). Contexto sociofamiliar de adolescentes ecuatorianos perpetradores de actividades delictivas. MEDISAN, 19(6). http://scielo.sld.cu/ scielo.php?script=sci_arttext\&pid=S1029-30192015000600007

Tijoux, M.E. (1995). Jóvenes pobres en Chile. Nadando en la modernidad y la exclusión. última década, (3), 1-9. http://www.cidpa.cl/wp-content/uploads/2013/06/3.3Tijoux.pdf

\section{(cc) BY}




\section{ANEXOS \\ Anexo 1. Formato de preguntas de la técnica grupo focal para evaluación por expertos}

\section{PROTOCOLO PARA LA EVALUACIÓN DE EXPERTOS}

Respetado(a) juez(a). Reciba un atento saludo y agradecimiento por su colaboración en la revisión y validación de las preguntas que se orientarán en el desarrollo del grupo focal en el proyecto "Factores socioambientales de la violencia urbana inmersos en los procesos de convivencia escolar de las Instituciones Educativas oficiales de las comunas 3, 7 y 10 de la ciudad de Pasto".

El objetivo general del estudio es: Comprender los factores socioambientales de la violencia urbana inmersos en los procesos de convivencia escolar de las Instituciones Educativas oficiales de las comunas 3, 7 y 10 de la ciudad de Pasto.

A continuación se presenta un formato que corresponde a las preguntas orientadoras del grupo focal, en los cuales están incluidos los ítems de la categoría FACTORES SOCIOAMBIENTALES DE LA VIOLENCIA URBANA, dividido en cuatro subcategorías. Le solicitamos marcar con Sí o con un NO de acuerdo a los siguientes criterios de calificación, y en caso de existir alguna observación por favor agregarla en el espacio Observaciones:

Claridad: El ítem es claro y comprensible.

Pertinencia: El ítem es adecuado en cuanto a la forma de su planteamiento, para el objetivo y la población a la cual se dirige.

Relevancia: El ítem es importante y ayuda a evaluar el atributo.

Redundancia: Lo que mide el ítem no es medido por ningún otro ítem.

Para la casilla de suficiencia (la cual hace referencia a si la cantidad de ítems por cada dimensión son representativos del universo de ítems posible para la misma), que se encuentra al pie de cada tabla, marque la opción Sí, si el número de ítems son representativos de su eje categorial, y marque la opción NO, si el número de ítems no son representativos de su eje categorial.

Muchas gracias por su colaboración. 
DATOS DEL EXPERTO:

\begin{tabular}{c}
\hline NOMBRE Y APELLIDOS \\
\hline PROFESIÓN \\
\hline FORMACIÓN ACADÉMICA (NIVEL MÁXIMO) \\
\hline CAMPOS O ÁREAS DE EXPERIENCIA PROFESIONAL \\
\hline TELÉFONO/ E-MAIL \\
\hline INSTITUCIÓN(ES) \\
\hline
\end{tabular}

REVISIÓN POR EXPERTOS DE PREGUNTAS ORIENTADORAS PARA EL GRUPO FOCAL

CATEGORÍA: FACTORES SOCIOAMBIENTALES DE LA VIOLENCIA URBANA INMERSOS EN PROCESOS DE CONVIVENCIA ESCOLAR

EJE CATEGORIAL 1: FACTORES SOCIOECONÓMICOS

Definición: El creciente fenómeno de la violencia urbana en América Latina se puede explicar a partir de factores como: la pobreza, la exclusión escolar y laboral, la disponibilidad de armas, la insatisfacción de las expectativas de las generaciones pobres, quienes miran en la violencia urbana el medio para construir su identidad y lograr satisfacer las expectativas de consumo impuestas por la sociedad, el consumo y tráfico de drogas donde se destacan prácticas de narcomenudeo y microtráfico (Briceño, 2002; Ministerio de Justicia y de Derecho, 2013, citado en Narváez y Pérez, 2017); estas actividades, entre otros aspectos, son un camino para conseguir un sustento económico (Narváez y Obando, 2017). Se considera además, que los altos niveles de desigualdad urbana dan paso a la construcción de barreras entre las comunidades marginadas y la clase dominante, lo que agudiza las desigualdades, desequilibrios sociales, políticos, económicos, naturales y culturales, derivando en la segregación espacial manifestada en comportamientos violentos y discriminatorios (Federación Internacional de Sociedades de la Cruz Roja y La Media Luna, 2010, citado en Narváez y Pérez, 2017; Briceño, 2007, citado en Narváez y Pérez, 2017). Por otra parte, el abandono gubernamental y el ordenamiento territorial suponeN procesos urbanísticos de exclusión y exclusividad donde la inversión estatal en mejoras estructurales se da para sectores residenciales de clase media-alta en cambio en las zonas de frontera y periferia se presenta una insuficiente planificación territorial lo que facilita la flagrancia y atrincheramiento de macroestructuras.

\begin{tabular}{|c|c|c|c|c|c|c|}
\hline ÍTEM & PREGUNTAS & CLARIDAD & PERTINENCIA & RELEVANCIA & REDUNDANCIA & OBSERVACIONES \\
\hline 1 & $\begin{array}{c}\text { ¿Qué formas o tipos de violencia obser- } \\
\text { van en su barrio? }\end{array}$ & & & & & \\
\hline 2 & $\begin{array}{l}\text { ¿Algunas formas o expresiones de violen- } \\
\text { cia que se viven en su barrio se repiten en } \\
\text { su colegio? ¿Cuáles? }\end{array}$ & & & & & \\
\hline 3 & $\begin{array}{l}\text { ¿Consideran que hay alguna relación } \\
\text { entre la ubicación de estos barrios con } \\
\text { algunas manifestaciones de violencia? }\end{array}$ & & & & & \\
\hline 4 & $\begin{array}{l}\text { ¿Cómo creen que los demás habitantes } \\
\text { de la ciudad los ven a ustedes por el } \\
\text { hecho de pertenecer a este sector? }\end{array}$ & & & & & \\
\hline 5 & $\begin{array}{l}\text { Por lo general, ¿en qué trabajan los } \\
\text { habitantes de sus barrios? }\end{array}$ & & & & & \\
\hline 6 & $\begin{array}{c}\text { ¿Han observado que en sus barrios se } \\
\text { realicen actividades ilegales o incorrec- } \\
\text { tas? ¿Cuáles? }\end{array}$ & & & & & \\
\hline 7 & $\begin{array}{l}\text { ¿Con qué fin creen que se realizan esas } \\
\text { actividades ilegales o incorrectas? }\end{array}$ & & & & & \\
\hline 8 & $\begin{array}{c}\text { ¿En este colegio han observado activida- } \\
\text { des parecidas a las que se presentan en } \\
\text { sus barrios? ¿Cuáles? }\end{array}$ & & & & & \\
\hline 9 & $\begin{array}{l}\text { ¿Es común que las personas porten algún } \\
\text { arma en sus barrios? ¿Por qué? }\end{array}$ & & & & & \\
\hline 10 & $\begin{array}{l}\text { ¿Algunos estudiantes traen armas al cole- } \\
\text { gio? ¿Cuáles? ¿Con qué propósito? }\end{array}$ & & & & & \\
\hline
\end{tabular}




\section{EJE CATEGORIAL 2: FACTORES POLÍTICOS DE LA VIOLENCIA URBANA}

Definición. Los estados que presentan altos niveles de violencia urbana se caracterizan por su incapacidad para prestar servicios y ofrecer seguridad; así pues, el contrato social entre el Estado y la sociedad es disfuncional. Cuando el contrato social funciona adecuadamente, el Estado moviliza ingresos públicos suficientes y ofrece seguridad y otros servicios esenciales, y los ciudadanos pagan sus impuestos, lo cual permite al Estado financiar la actividad destinada a producir esos bienes públicos. Por ende, la legitimidad del Estado se ve fortalecida y la estabilidad reforzada. Cuando el Estado no satisface las expectativas de los ciudadanos, esta legitimidad se debilita. En algunos países, las élites no están dispuestas a financiar los programas sociales.

Los cambios y la diversidad de necesidades de movilidad de la fuerza laboral urbana permanentemente crean nuevas demandas y ajustes en términos de políticas de infraestructura y por lo general los programas diseñados para aliviar las nuevas necesidades de movilidad no suelen dar las respuestas requeridas con la prontitud deseada, lo que genera violencia dentro de las ciudades, donde las personas no satisfacen algunas de sus necesidades, y se ven obligadas a recurrir a otro tipo de actividades como el pandillismo, el microtráfico, etc.

\begin{tabular}{|c|c|c|c|c|c|}
\hline ÍTEMS & PREGUNTAS & CLARIDAD & PERTINENCIA RELEVANCIA & REDUNDANCIA & OBSERVACIONES \\
\hline 1 & $\begin{array}{c}\text { ¿Podrían describir la seguridad en sus } \\
\text { barrios? }\end{array}$ & & & & \\
\hline 2 & $\begin{array}{c}\text { ¿De qué manera el gobierno les garantiza } \\
\text { seguridad y protección a los habitantes en } \\
\text { sus barrios? }\end{array}$ & & & & \\
\hline 3 & $\begin{array}{c}\text { ¿Qué instituciones del Estado hacen } \\
\text { presencia en sus barrios para garantizar la } \\
\text { protección de los habitantes? }\end{array}$ & & & & \\
\hline 4 & $\begin{array}{c}\text { ¿Cómo se cuidan o protegen a sí mismos } \\
\text { de la violencia o la inseguridad en su } \\
\text { barrio? }\end{array}$ & & & & \\
\hline 5 & $\begin{array}{l}\text { Si experimentan alguna forma de violen- } \\
\text { cia en el colegio, ¿cómo actúan? }\end{array}$ & & & & \\
\hline \multirow[t]{2}{*}{6} & $\begin{array}{l}\text { ¿Han observado alguna situación de vio- } \\
\text { lencia que se presenta tanto en el barrio } \\
\text { como en el colegio? ¿Cuál? }\end{array}$ & & & & \\
\hline & & Suficiencia Sí_ & No_ & & \\
\hline & EJE CATEGORIAL 3: FAC & STORES RELAC & CIONADOS CON EL CONTROL & TERRITORIAL & \\
\hline
\end{tabular}

De acuerdo con Angarita (2012) el control territorial es entendido como el control del espacio físico en donde el grupo ejerce su poder de manera permanente para generar dinámicas de territorialización o de apropiación del territorio y la conservación del grupo ilegal, realizando actuaciones para la protección y perpetuación de este. Para Pérez (2013) el control territorial es un proceso que se desarrolla a través de vías de hecho para la apropiación y el ejercicio del poder, que se nutre y reproduce aprovechando las fracturas generadas por la inoperancia del Estado y el financiamiento de estructuras macrodelincuenciales, trayendo como efecto, la reducción de los espacios, medios y formas de participación, así como, la seguridad en salud y educación, al impedir la movilidad de las personas, favoreciendo el establecimiento de fronteras territoriales ilegales, las cuales llevan a la delimitación y demarcación de lugares que indican espacios de confrontación violenta, de control no disputado y de prohibición de la libre circulación.

\begin{tabular}{|c|c|c|c|c|c|c|}
\hline ÍTEMS & PREGUNTAS & CLARIDAD & PERTINENCIA & RELEVANCIA & REDUNDANCIA & OBSERVACIONES \\
\hline 1 & $\begin{array}{l}\text { ¿Han identificado si en sus barrios hay } \\
\text { pandillas, grupos delincuenciales o algo } \\
\text { similar? ¿Qué hacen estos grupos? }\end{array}$ & & & & & \\
\hline 2 & $\begin{array}{l}\text { ¿Cuáles son las características particula- } \\
\text { res que identifican a los integrantes de } \\
\text { esos grupos? }\end{array}$ & & & & & \\
\hline 3 & $\begin{array}{l}\text { ¿Qué lugares del barrio suelen ocupar o } \\
\text { transitar los integrantes de estos grupos? }\end{array}$ & & & & & \\
\hline 4 & $\begin{array}{l}\text { ¿Hay momentos del día u horas específi- } \\
\text { cas en las que no se pueda transitar por } \\
\text { algunos lugares de sus barrios? }\end{array}$ & & & & & \\
\hline
\end{tabular}


¿Hay algún lugar en sus barrios por

5 donde no pueda transitar todo el mundo?

$$
\text { ¿Cuál? }
$$

6

¿Quiénes tienen el control y pueden estar o transitar por esos lugares del barrio?

7

¿De qué forma estas personas controlan dichos espacios o lugares?

8

¿Qué tipo de actividades se realizan en esos lugares?

Al igual que en sus barrios, ¿en su colegio

han visto si hay también lugares por donde es peligroso andar?

¿Qué suele suceder en esos lugares del

colegio y quienes generalmente van por ahí?

¿Ven alguna semejanza o parecido de lo que pasa en su barrio, a lo que pasa en el colegio con respecto a la existencia de estos lugares?

Suficiencia Sí___ No

\section{EJE CATEGORIAL 4: FACTORES FAMILIARES DE LA VIOLENCIA URBANA}

Definición: La familia es el primer contacto que el sujeto tiene con la sociedad, por tanto las pautas de crianza y los patrones que se instauren al interior del hogar son determinantes en el desarrollo de las conductas de los individuos, es así como en diversos estudios se ha encontrado que las conductas antisociales que puedan presentar los niños o adolescentes se deben a un clima familiar caracterizado por una pobre cohesión y falta de normas claras; patrones de comunicación poco fluidos o comunicación rígida; una pobre satisfacción familiar; presencia de violencia transgeneracional; que los hijos experimenten violencia entre sus padres o experimenten castigos físicos; estilos educativos erróneos de los padres como la legitimación del castigo o maltrato infantil; y que los padres consuman drogas o alcohol (Rivera \& Cahuana, 2016). Asimismo Gutiérrez, Campo y Portillo (2015) plantean que aquellos jóvenes que han crecido con una sola figura paterna, tienen una mayor probabilidad de desarrollar conductas antisociales, dado que presentan menos supervisión y control, viven en un ambiente familiar hostil, son víctimas de violencia física y presentan más inestabilidad emocional que los jóvenes que crecieron con ambos padres.

\begin{tabular}{|c|c|c|c|c|c|c|}
\hline ÍTEMS & PREGUNTAS & CLARIDAD & PERTINENCIA & RELEVANCIA & REDUNDANCIA & OBSERVACIONES \\
\hline 1 & $\begin{array}{c}\text { ¿Cómo describe las relaciones familiares } \\
\text { que se dan en su hogar? }\end{array}$ & & & & & \\
\hline 2 & $\begin{array}{l}\text { ¿Qué comportamientos violentos o agre- } \\
\text { sivos se dan a veces en sus hogares? }\end{array}$ & & & & & \\
\hline 3 & $\begin{array}{l}\text { De los anteriores comportamientos que } \\
\text { observan en sus hogares, ¿̇algunos se } \\
\text { repiten en la escuela? ¿Cuáles? }\end{array}$ & & & & & \\
\hline 4 & $\begin{array}{c}\text { ¿Cómo resuelven los conflictos en sus } \\
\text { hogares? }\end{array}$ & & & & & \\
\hline 5 & ¿Qué valores les enseñan en sus casas? & & & & & \\
\hline 6 & $\begin{array}{c}\text { ¿Qué valores fomentados en sus familias } \\
\text { les cuesta practicar? }\end{array}$ & & & & & \\
\hline 7 & $\begin{array}{c}\text { ¿Cómo actúan sus padres si ustedes no } \\
\text { cumplen las normas de la casa o si se } \\
\text { portan mal? }\end{array}$ & & & & & \\
\hline 8 & $\begin{array}{l}\text { En las familias de sus barrios, ¿qué com- } \\
\text { portamientos violentos han observado? }\end{array}$ & & & & & \\
\hline & & Suficiencia Sí_ & No_ & & & \\
\hline
\end{tabular}




\section{Anexo 2. Formato de preguntas para entrevista semiestructurada}

\section{FACTORES SOCIOECONÓMICOS}

1. ¿Qué formas o tipos de violencia se observan en este sector?

2. ¿Considera que hay alguna relación entre la ubicación de estos barrios con algunas manifestaciones de violencia?

3. ¿Cómo cree que los demás habitantes de la ciudad los ven a ustedes por el hecho de pertenecer a este sector?

4. Por lo general, ¿̇en que trabajan los habitantes de este sector?

5. ¿Ha observado que en este sector se realicen actividades ilegales o incorrectas? ¿Cuáles?

6. ¿Con qué fin cree que se realizan esas actividades ilegales o incorrectas?

7. ¿En el colegio ha observado actividades parecidas a las que se presentan en este sector? ¿Cuáles?

8. ¿Es común que las personas porten algún tipo de arma en este sector? ¿Por qué?

\section{FACTORES POLÍTICOS}

1. ¿Podría describir la seguridad en este sector?

2. ¿De qué manera el gobierno les garantiza seguridad y protección a los habitantes de este sector?

3. ¿Qué instituciones del Estado hacen presencia en este sector para garantizar la protección de los habitantes?

4. ¿Cómo se cuidan o se protegen a sí mismos de la violencia o la inseguridad en este sector?

5. ¿Cómo actúan los jóvenes cuando se presentan situaciones de violencia al interior o alrededor del colegio?

\section{FACTORES DE CONTROL TERRITORIAL}

1. ¿Ha identificado si en este sector hay pandillas, grupos delincuenciales o algo similar? ¿Qué hacen estos?

2. ¿Cuáles son las características particulares que identifican a los integrantes de estos grupos?

3. ¿Qué lugares de este sector suelen ocupar o transitar los integrantes de estos grupos? 
4. ¿Hay momentos del día u horas específicas en las que no se pueda transitar por algunos lugares de este sector?

5. ¿Hay algún lugar en este sector por donde no pueda transitar todo el mundo? ¿Cuál?

6. ¿Quiénes tienen el control y pueden estar o transitar por esos lugares de este sector?

7. ¿De qué forma estas personas controlan dichos espacios o lugares?

8. ¿Qué tipo de actividades se realizan en estos lugares?

9. Al igual que en este sector, ¿en el colegio ha visto si hay también lugares por donde es peligroso andar?

10. ¿Qué suele suceder en esos lugares del colegio y quiénes generalmente van por ahí?

11. ¿Ve alguna semejanza o parecido de lo que pasa en este sector, a lo que pasa en el colegio con respecto a la existencia de estos lugares?

\section{FACTORES FAMILIARES}

1. ¿Qué comportamientos se dan en los hogares de este sector?

2. De los anteriores comportamientos que observa en los hogares del sector, ¿algunos se repiten en la escuela? ¿Cuáles?

3. En las familias de estos barrios, ¿qué comportamientos violentos ha observado? 
